\title{
Picosecond X-Ray Generation Through Thomson Scattering
}

\author{
Salim H. Al-Shamma Muzahim I. Azawi \\ Lubna H. Ismail \\ Department of Physics / College of Education \\ University of Mosul
}

Received

21 / 10 / 2009
Accepted

16 / 02 / 2010

الظلاصة

إن توليد الأشعة المينية ذات نبضة قصيرة من خلال لطسطارة ثومسون لها فائية لـ ـيس

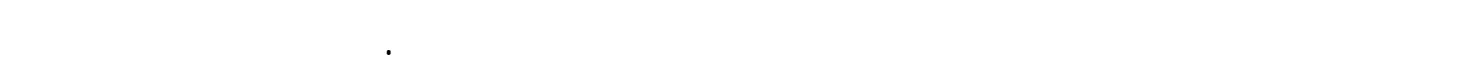

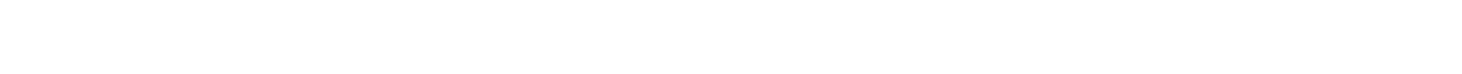

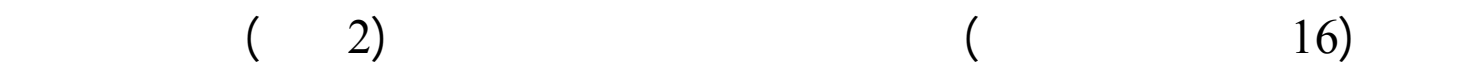

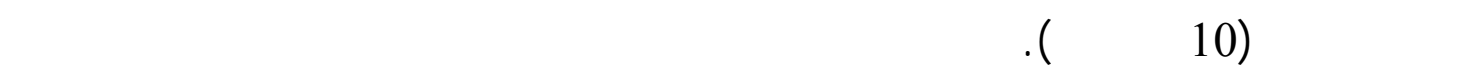

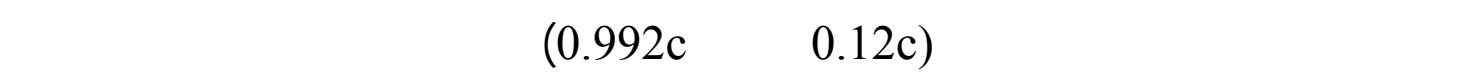

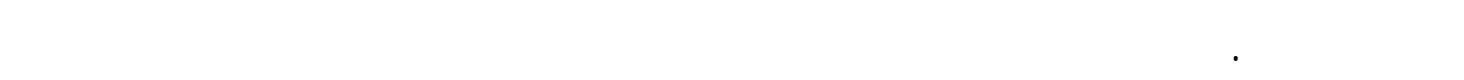

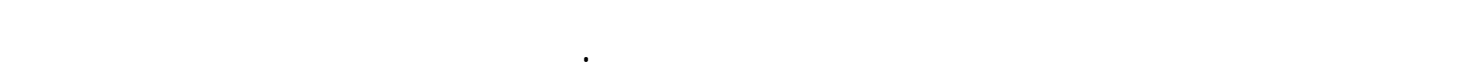

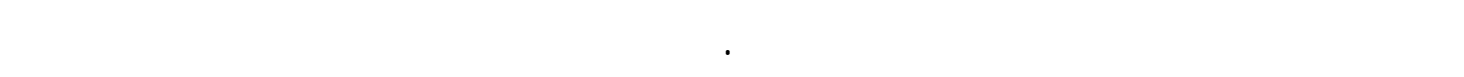
هنه المديلت في هذه الدرلسة.

\section{Abstract}

Short pulse $\mathrm{x}$-ray generation which is produced through Thomson scattering is of interest not only to the basic collision physics, but also used in various research fields. In this work, some theoretical results have been obtained on Thomson scattering between $(16 \mathrm{MeV})$ relativistic electron energy and laser pulse energy (2J) of pulse duration (10ns). The differential cross sections of Thomson scattering have been determined between $0.1 \mathrm{c}$ up to $0.99 \mathrm{c}$ electron energies through different $\mathrm{x}$-ray emitting angles. A relation between the differential cross sections and incident electron energies is established at an $\mathrm{x}$-ray emitting angle equal to zero. In addition, $\mathrm{x}$-ray pulse length over different scattering angles 
have been determined. To our work knowledge, no experimental data available of this work for comparison.

\section{Introduction}

The generation of $\mathrm{x}$-ray by scattering between relativistic electrons and laser light was proposed in 1960[1]. the angstrom scale wavelengths of x-ray have the advantages compared to other light sources, such as (Ti:Sp laser) of the wavelength $800 \mathrm{~nm}$, in that they give excellent resolution, capable of penetration of atoms, exciting deeper core electrons capable of imaging surfaces and standing crystalline structure. Thomson scattering is one of the most promising approaches to ultra short pulsed $\mathrm{x}-$ rays [2]. A tunable ultra short pulse x-ray sources of pulse duration (in nanosecond and femtosecond) are being widely used in various research fields such as solid state physics, material science, atomic and molecular physics with femtosecond optical and x-ray pulses, ultra fast photo chemical reactions, medical imaging, and industrial applications [3]. Researches on the Thomson scattering x-rays sources received increased interest in recent years[4,5,6,7 and 8].

In this work, theoretical results on Thomson scattering between relativistic electron beam and short pulse laser have been presented, such as differential cross sections of Thomson scattering have been estimated for a wide range of x-rays emitting angles, polar plots of differential cross sections against $\mathrm{x}$-rays emitting angles have been estimated. Furthermore, the results of $\mathrm{x}$-rays pulse length for a different scattering angles have been also determined.

\section{Basic Principle of Inverse of Compton (Thomson Scattering)}

Thomson Scattering of photon by an electron at rest, strictly only applied at low photon energy, i.e., when ( $\left.h v<<\mathrm{m}_{0} \mathrm{c}^{2}\right)$ (Compton effect). if the photon energy is comparable or greaten them electron rest energy i.e $\left(h v>>m_{0} c^{2}\right)$ relativistic effect must be taken in to account. An important case arise when the electron no longer to be at rest. energy is transferred from the electron to photon, i.e., it is opposite of Compton scattering in which the photon transfer energy to the electron [1].The generation of the X-rays in the inverse scattering where abeam of accelerated electrons are collided with a burst of photons in vacuum. Below is a diagram of the interaction configuration. 


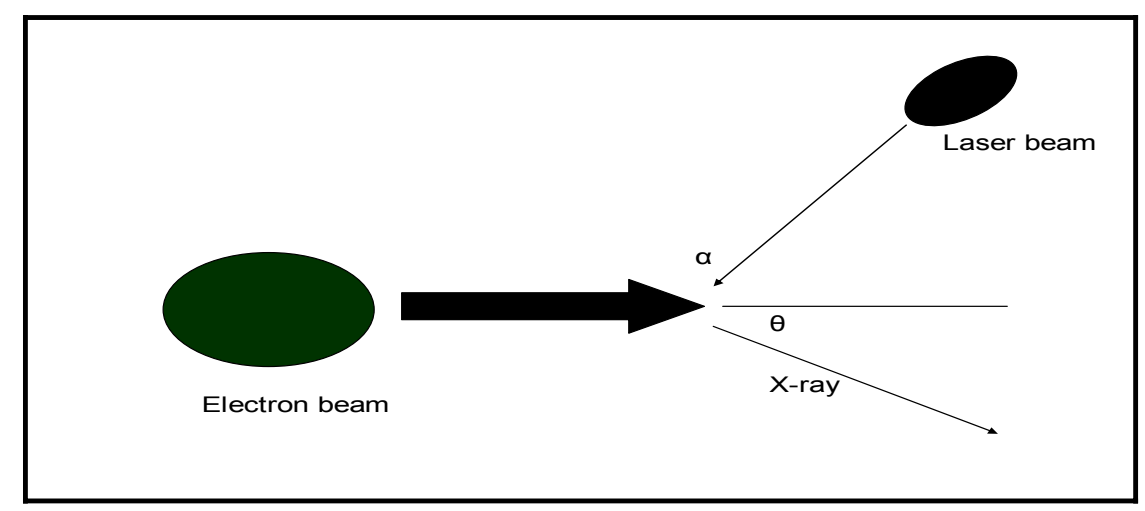

Fig(1)Diagram of interaction configuration

In this process, the electrons oscillate under the influence of the electric field of photons. The oscillating electrons emit photon soft x-ray region.The accelerator for electrons operate at repetition rate from a few $\mathrm{Hz}$ to $120 \mathrm{~Hz}$. The laser needed to produce inverse Compton scattering have the following properties[9].

1- High number of photon per unite time.

2- Short pulse duration (a few Ps).

3- Low oscillator jitter.

4- High beam stability.

5- High beam profile.

\section{Theory}

The theoretical background of Thomson scattering between relativistic electron beam and short pulse laser light is reviewed else where[2,3,6,9]. In Thomson scattering the differential is given [1]

$$
\frac{d \delta_{T}}{d \Omega}=\frac{1}{2} r_{o}^{2}\left(1+\cos ^{2} \alpha\right)---(1)
$$

Where $r_{o}$ is the classical radius of electron, $\alpha$ is the scattering angle.

The differential cross section of the relativistic Thomson scattering is given by[6].

$$
\frac{d \Sigma}{d \Omega}=r_{o}^{2}\left(1+\mathrm{B}^{2}\right)\left(1+\cos ^{2} \theta_{x}\right)-4 \mathrm{~B} \cos \theta_{x} / 2 \gamma^{2}\left(1-\mathrm{B} \cos \theta_{x}\right)^{2}---(2)
$$

Where $\theta_{\mathrm{x}}$ is the emitting angle of $\mathrm{x}$-ray, $\mathrm{r}_{\mathrm{o}}$ is the classical radius of electron, $\mathrm{B}=\mathrm{v} / \mathrm{c}$ where $\mathrm{v}$ is the velocity of electron and $\mathrm{c}$ is the velocity of Light.

The pluse length of the generated $x$-ray is given by[6]

$\delta=\delta_{e z} \sqrt{(1-\cos \alpha)\left(\delta_{e z}^{2}+\delta_{L z}^{2}\right)+\left(1+\cos (\alpha) \delta_{L z}^{2)}\right.} / c \sqrt{(1-\cos \alpha)\left(\delta_{e z}^{2}+\delta_{L z}^{2}\right)-(1+\cos \alpha)\left(\delta_{e z}^{2}+\delta_{L z}^{2}\right)}$

Where $\alpha$ is the scattering angle, $\delta_{\mathrm{ez}}$ is the electron rms size for electron beam, $\delta_{\mathrm{Lz}}$ is the laser rms size for laser beam and $\mathrm{c}$ is the velocity of light. 


\section{Results and discussions}

The results below is a theoretical simulations are resulted through Thomson scattering.In this process an electron beam of relativistic energy $(16 \mathrm{Mev})$ of microbunch duration of $(35 \mathrm{ps})$ with $\mathrm{rms}$ beam radius of $(1.25 \mathrm{~mm})$ is collided with laser beam [6]. The laser Nd: YAG of wavelength $(1.064 \mu \mathrm{m})$, pulse duration (10ns), pulse energy (2J) and of focal radius $(0.5 \mathrm{~mm})$ form was used in the present study fig. (2) show the classical behavior of Thomson differential cross section against the scattering angle (alpha) according to equation (1). From this fig. we can notice the periodicity behavior of the Thomson differential cross section due to the influence of $\left(\cos ^{2} \theta\right)$ factor in eq. (1). Fig (3) Shows the behavior of the differential cross section of Thomson scattering against a wide range of $x$-rays emitting angle (theta) and wide range of impact velocity of electron (nearly the classical limit). From the fig ( $\mathrm{v}=0.1 \mathrm{c}$ and $0.2 \mathrm{c}$ ), we can notice that the differential cross section depend on x-ray emitting angle. For larger value of electron beam, the differential cross section is independent on the emitting angle (see fig 4). In order to illustrate the effect of large value of emitting energy $(\mathrm{v} \sim \mathrm{c})$, the differential cross section had a larger value compare to. Fig(5) on the other band that the differential cross section decreases rapidly with the $\mathrm{x}$ ray emitting angle and approaches to zero at an angle (0.02) radians Fig(6). Illustrate the effect of the electron beam velocity on the differential cross section. In other word the dependence of differential cross section upon the electron beam energy rather than the electron velocity is shown in.Fig (7) as shown from this figure that the threshold value to generate $\mathrm{x}$-ray pulse is about $(0.5 \mathrm{Mev})$. polar plots of the differential cross sections for different values of electron velocities and $\mathrm{x}$ ray emitting angle have shown is Fig. (8,9,10 and 11). Fig (12) shows $x-$ rays picosecond pulse generation the scattering angle (theta) for different values of longitudinal coordinate ( $\mathrm{sig} \mathrm{ze}=1 \mathrm{~mm}, 2 \mathrm{~mm}$ and $5 \mathrm{~mm}$ ). We can notice from this figure that the pulse length decreases with increasing (sig ze) The projection of x-ray picosecond pulses for these value of sig ze had shown is Fig. (12b, 12c and 12d). The projection is concentric for $(\operatorname{sig} \mathrm{ze}=1 \mathrm{~mm})$. And deviated for other values of sig $\mathrm{ze}(3 \mathrm{~mm}$ and $5 \mathrm{~mm})$.

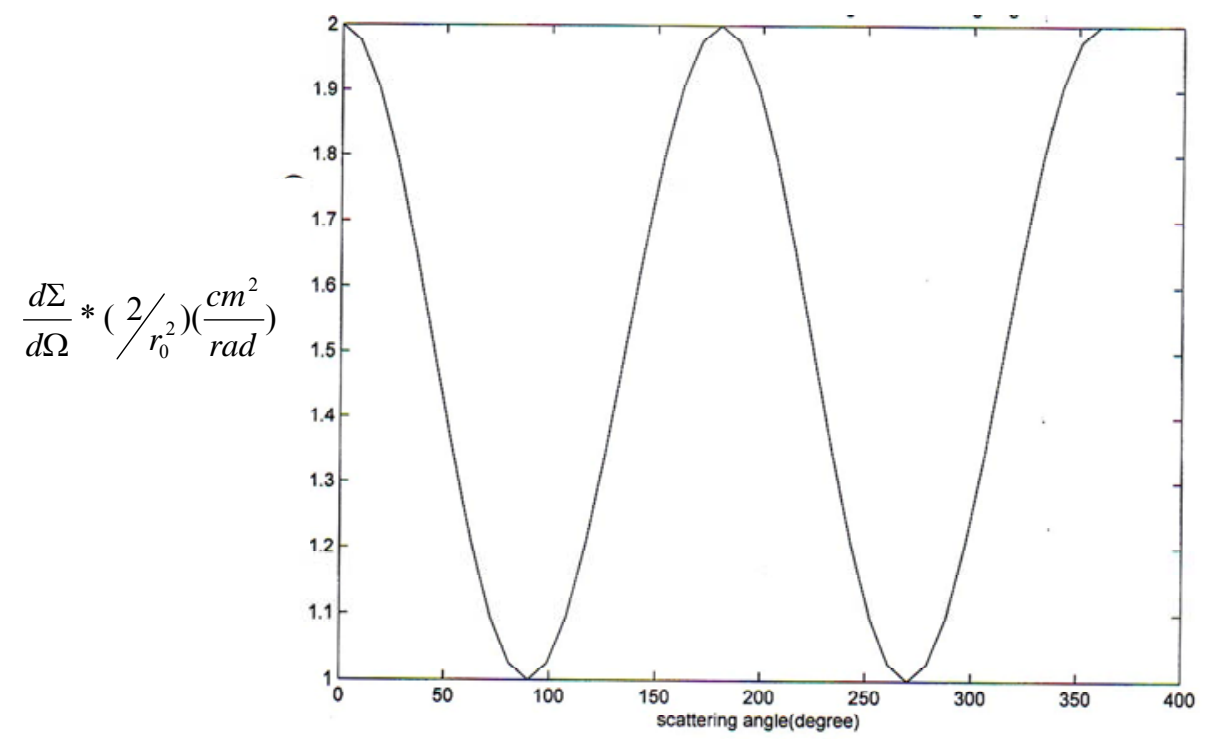

Fig(2)Thomson differential cross section against scattering angle 


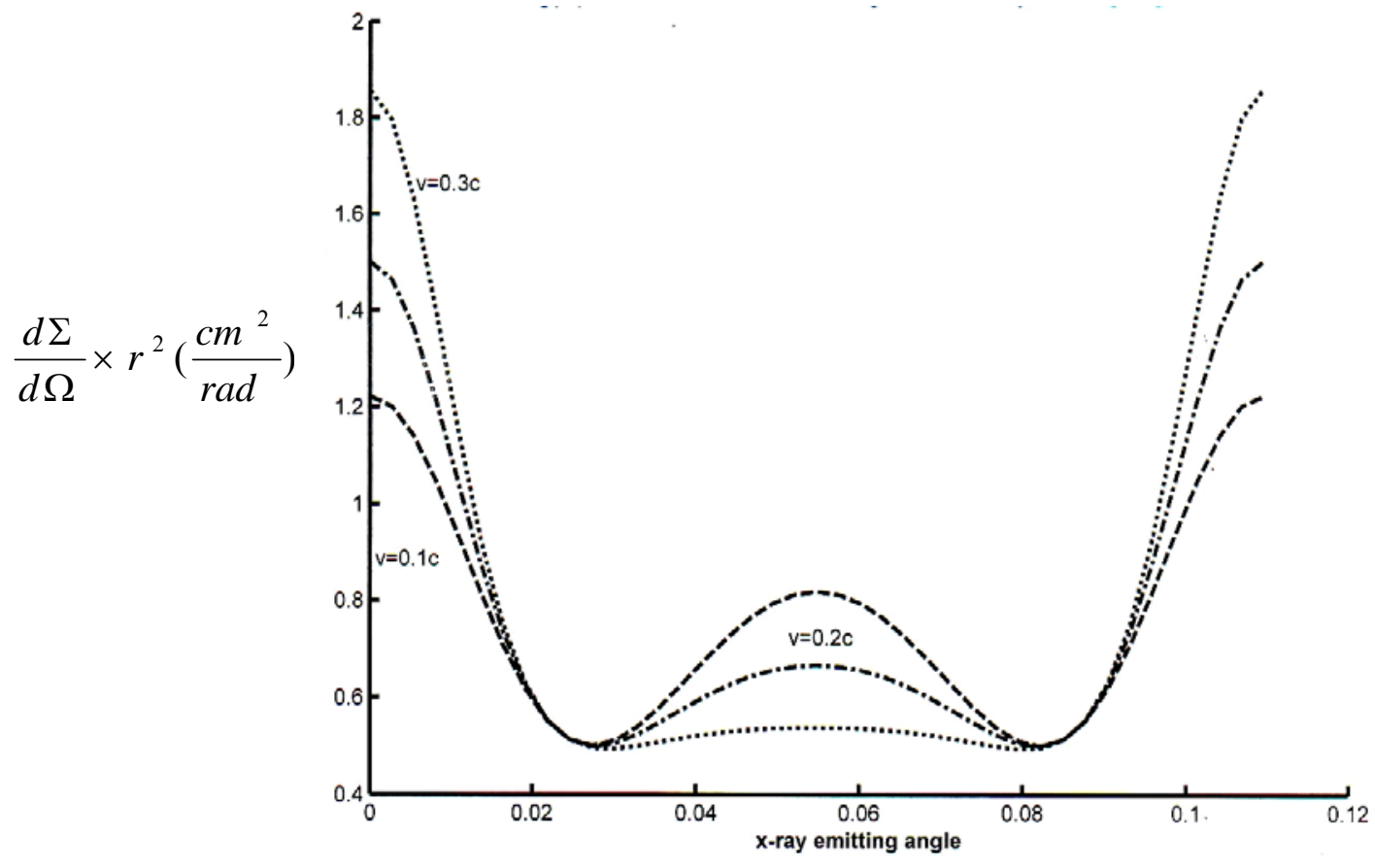

Fig(3) differential cross section against the $x$-ray emitting angle

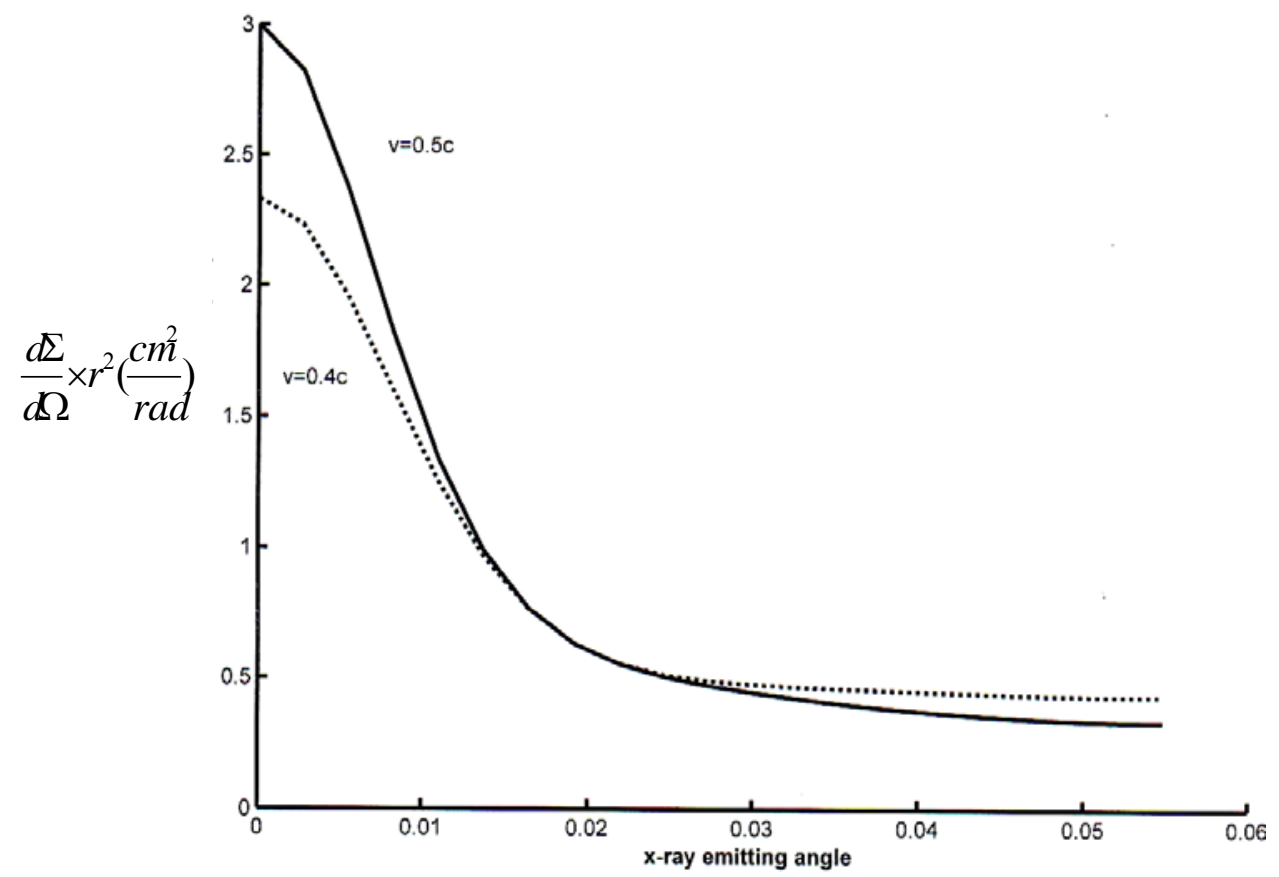

Fig (4) Differential cross section against x-ray emitting angle 


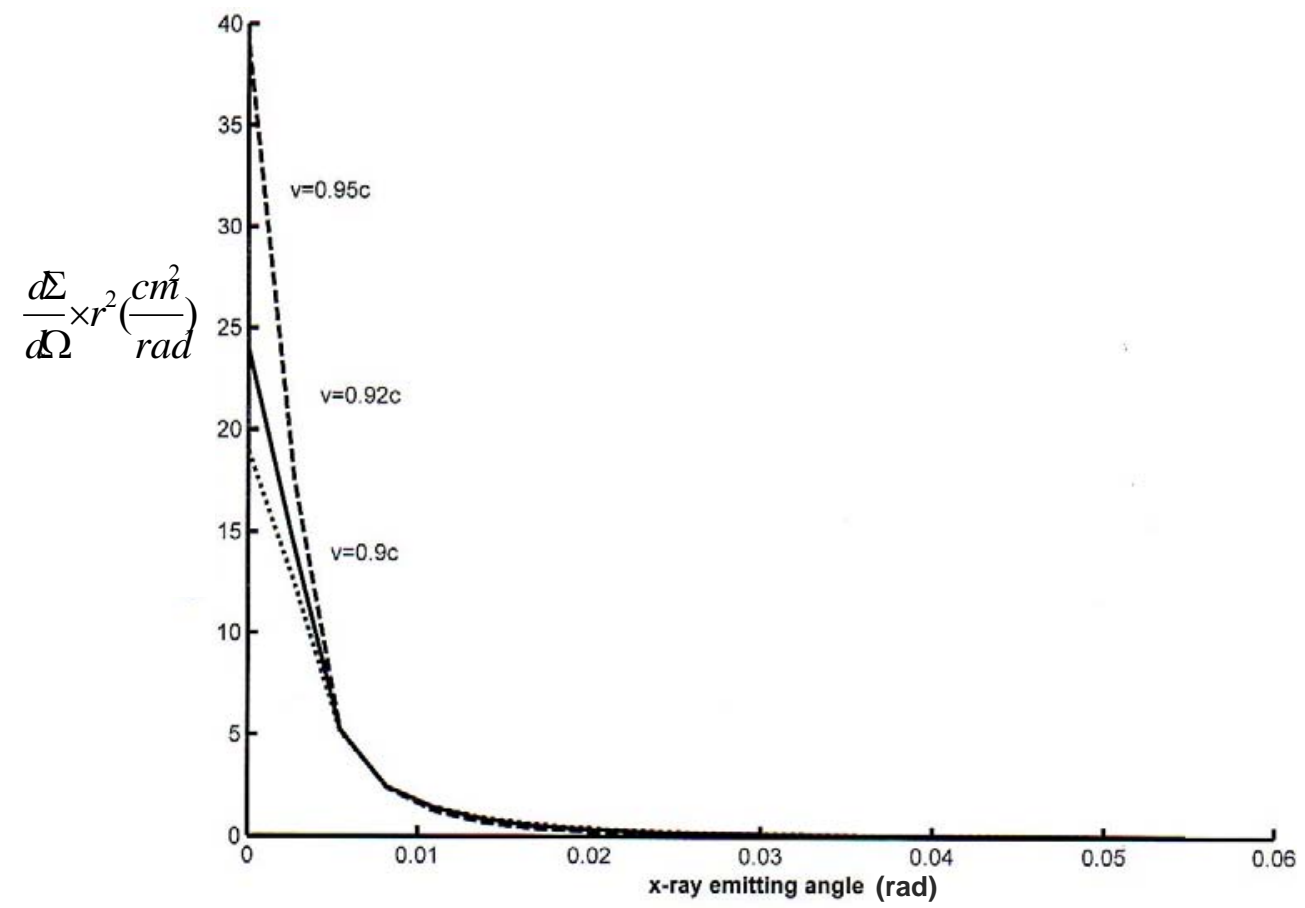

Fig (5) Differential cross section against x-ray emitting angle

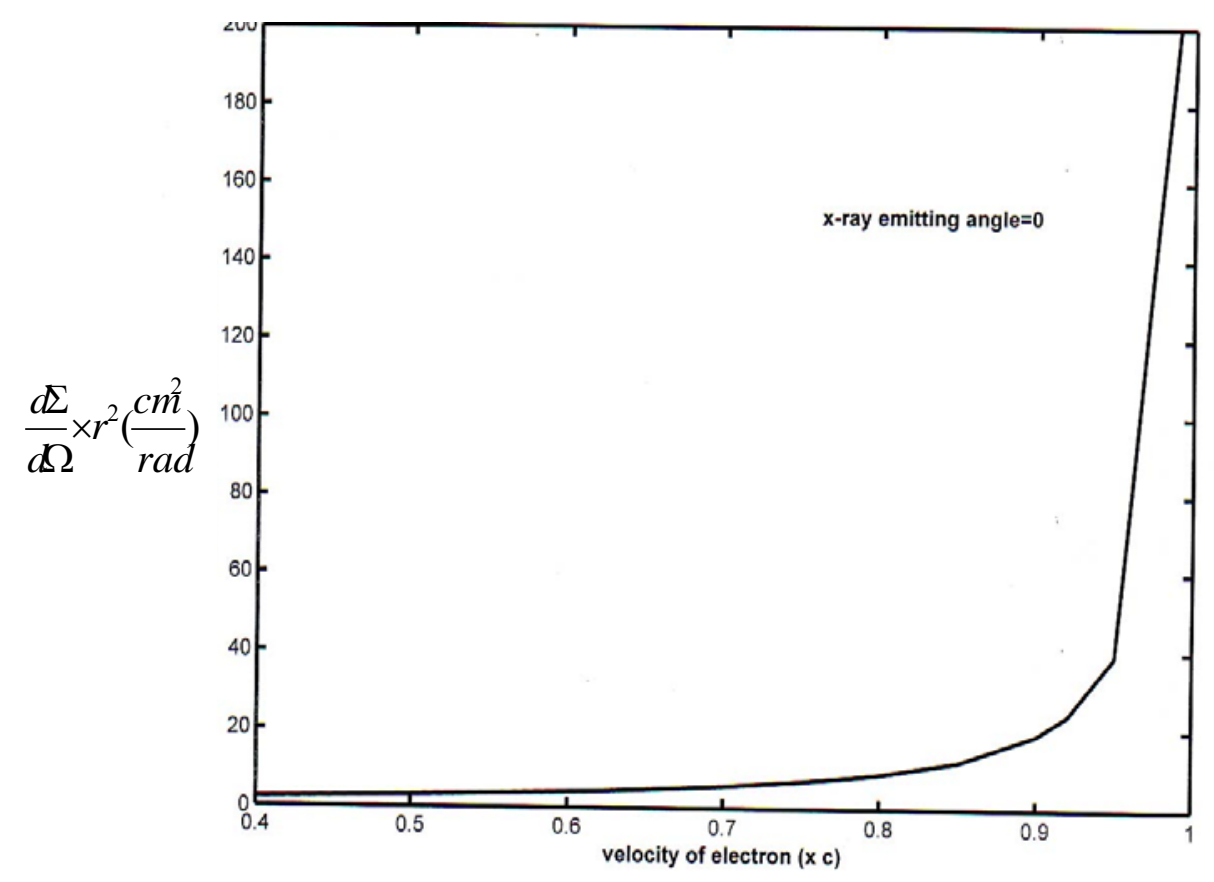

Fig (6) Differential cross section against electron velocity 
Salim H. Al-Shamma \& Muzahim I. Azawi \& Lubna H. Ismail

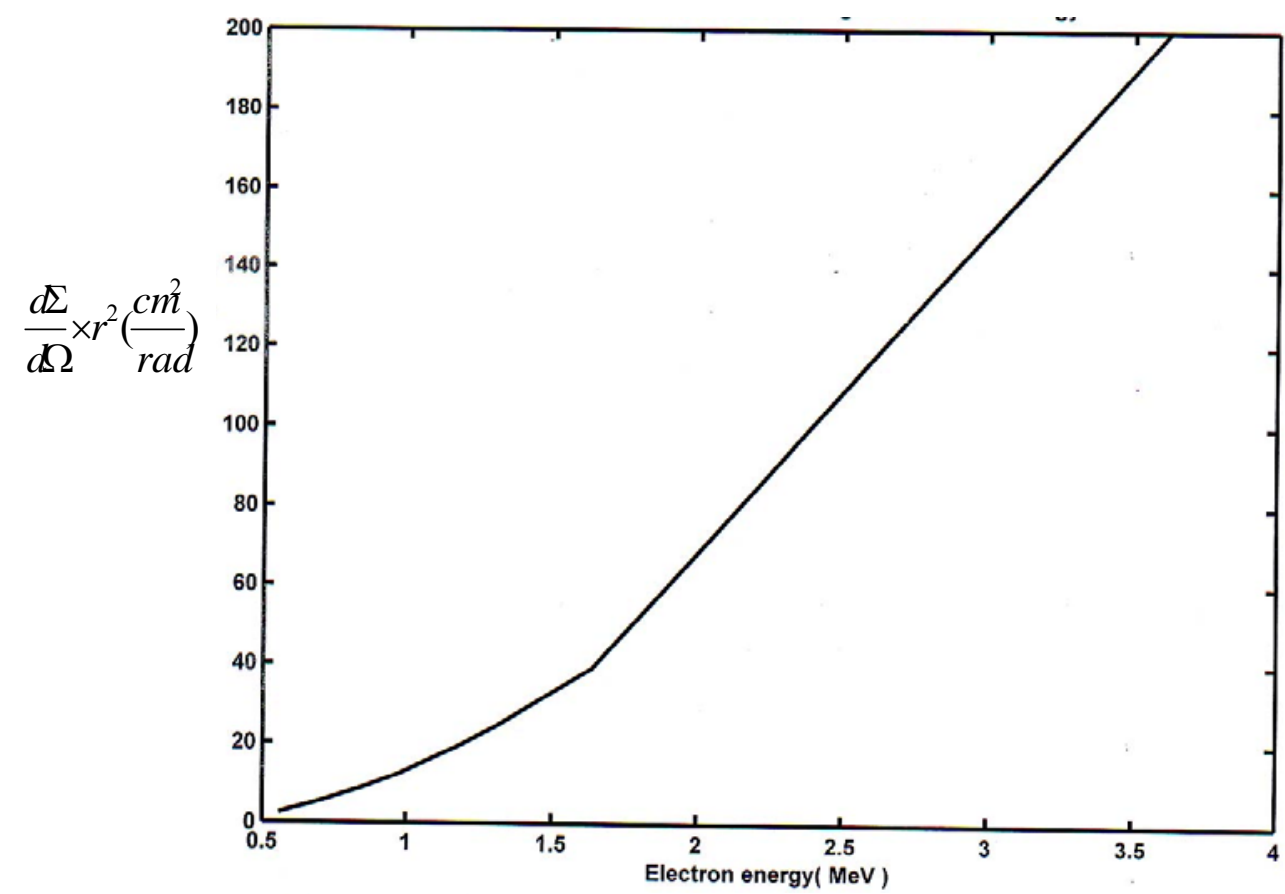

Fig (7) Differential cross section against electron energv

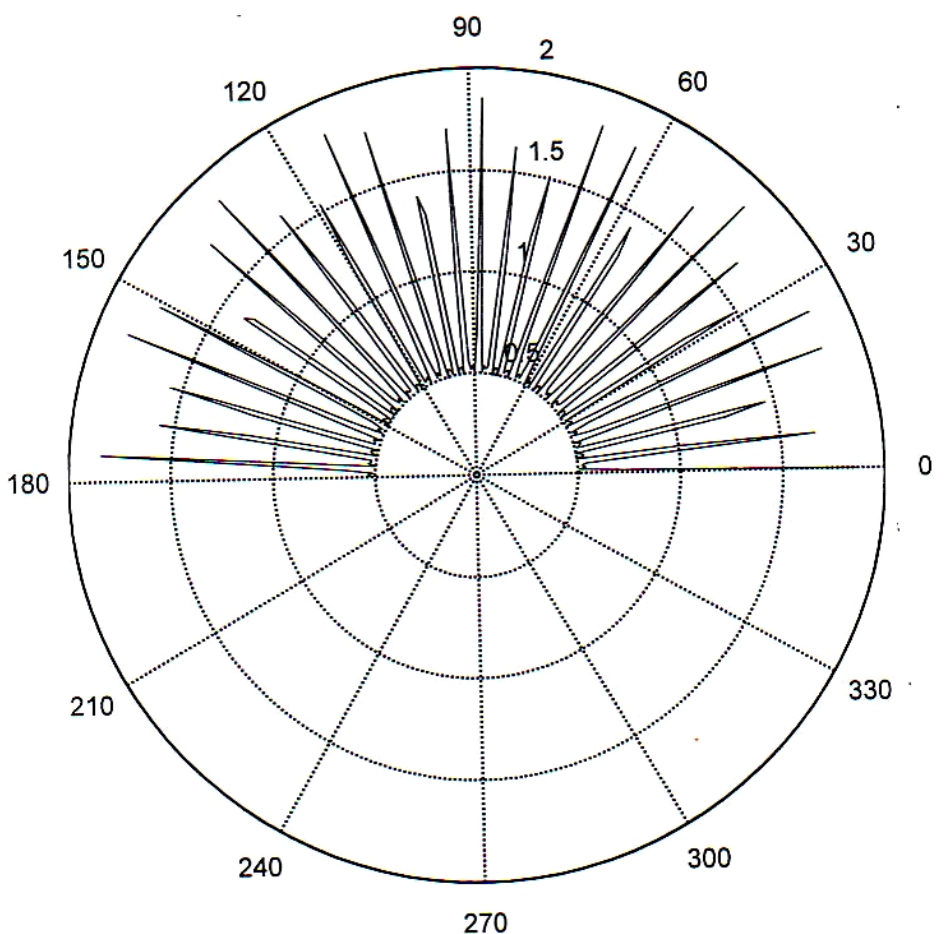

Fig (8) Polar plot of differenial cross section( ${ }^{*}{ }^{2}{ }^{2}{ }^{2}$ ) at $v=0.3 \mathrm{c}$ 


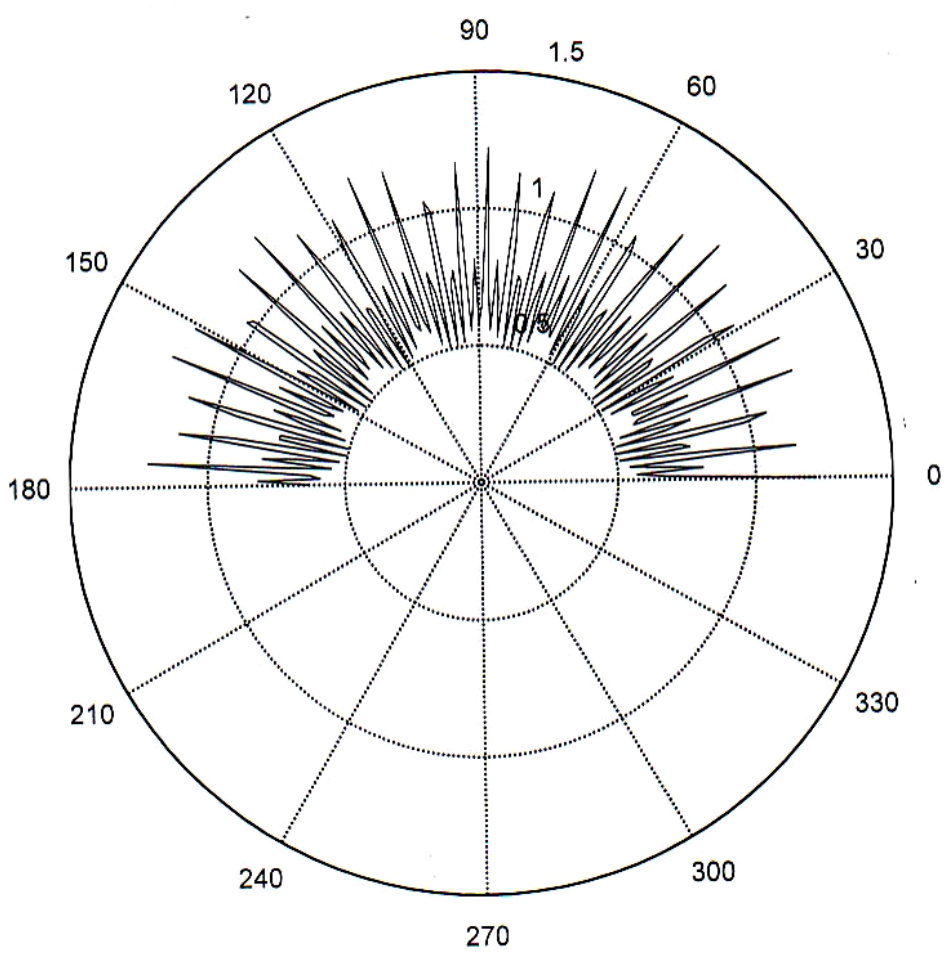

Fig (9) Polar plot of differential cross section $\left({ }^{\star}\right.$ ro. $\left.{ }^{5}\right)$ at $v=0.7 \mathrm{c}$

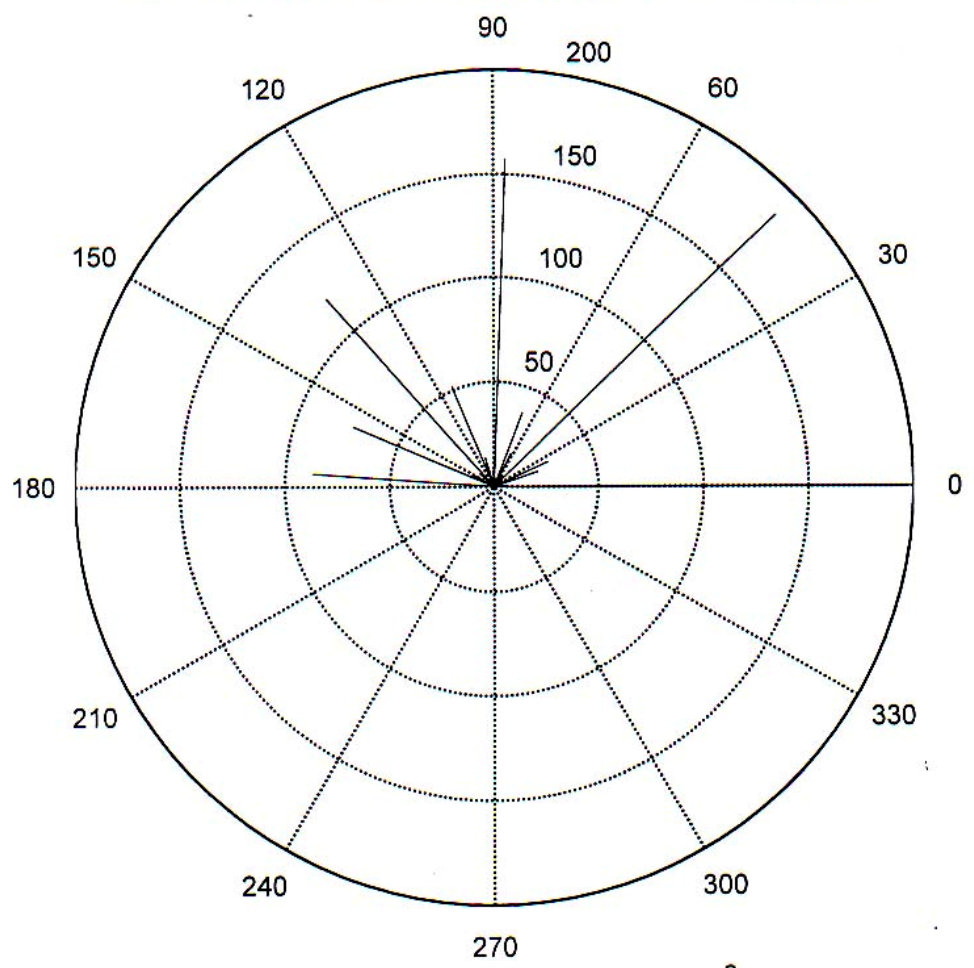

Fig (10)Polar plot of differential cross section $\left({ }^{*}{ }^{*}{ }^{2}{ }^{2}\right)$ at $v=0.99 c$ 


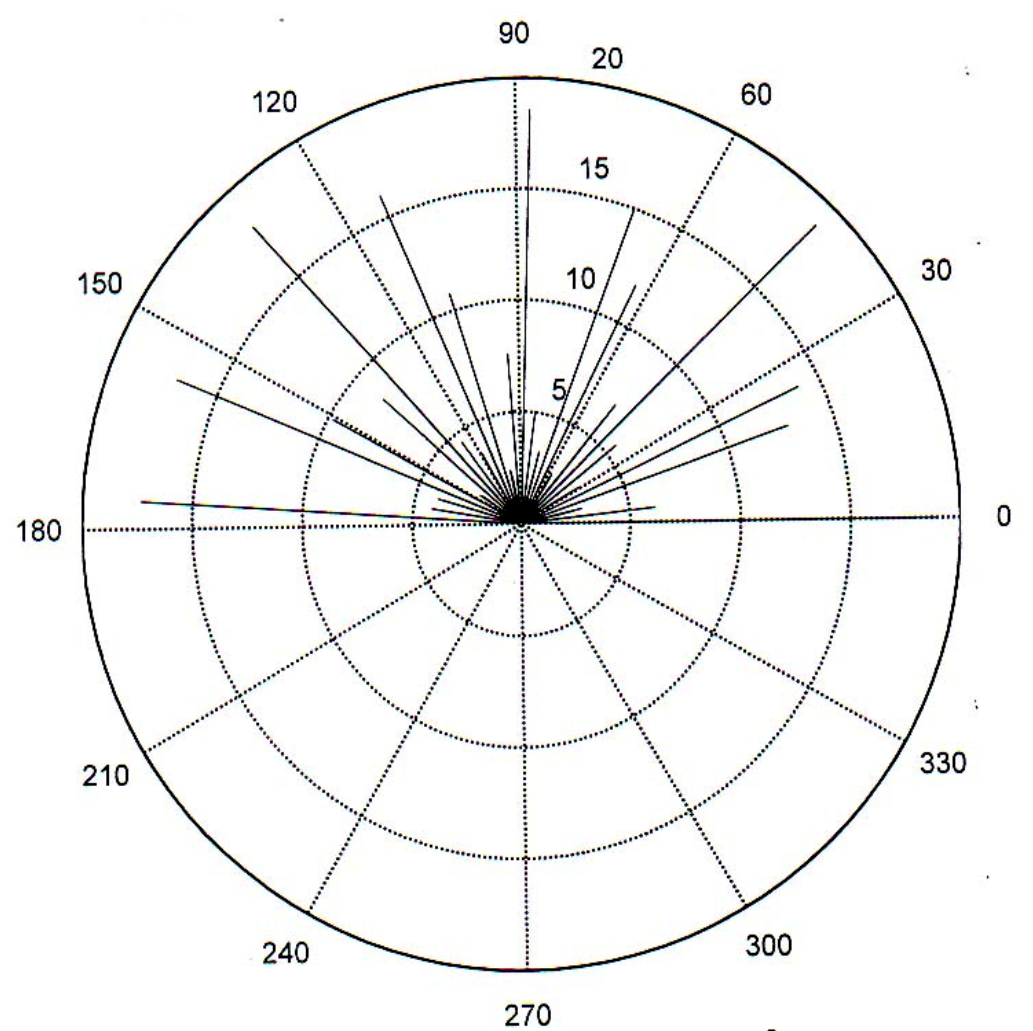

Fig (11)Polar plot of differential cross section(" ${ }^{*}$ ro. $\left.{ }^{2}\right)$ at v $=0.9 \mathrm{c}$

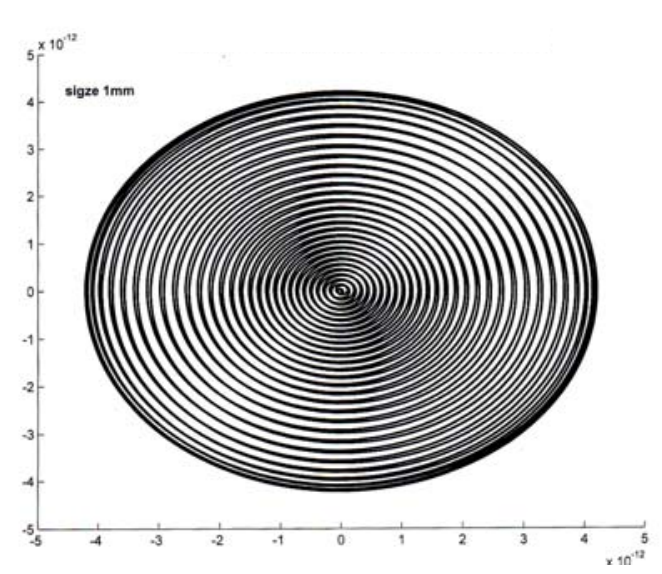

Fig 12\$Polar plot of pulse width against the angle alpha

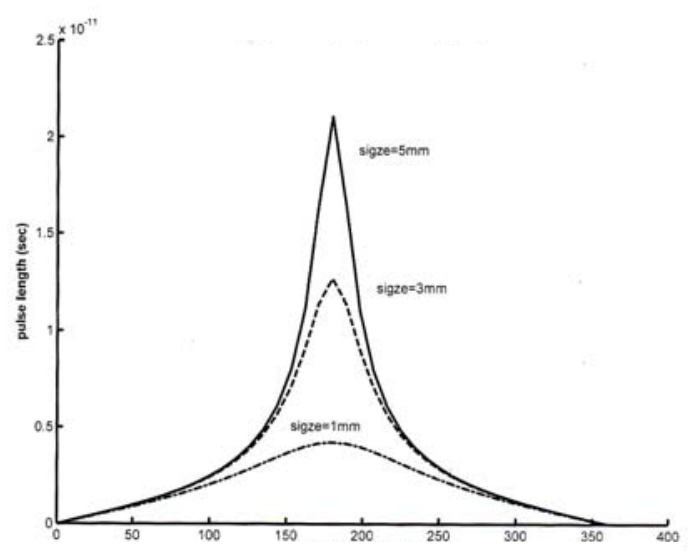

Fig12d Pulse length against the angle alpha 

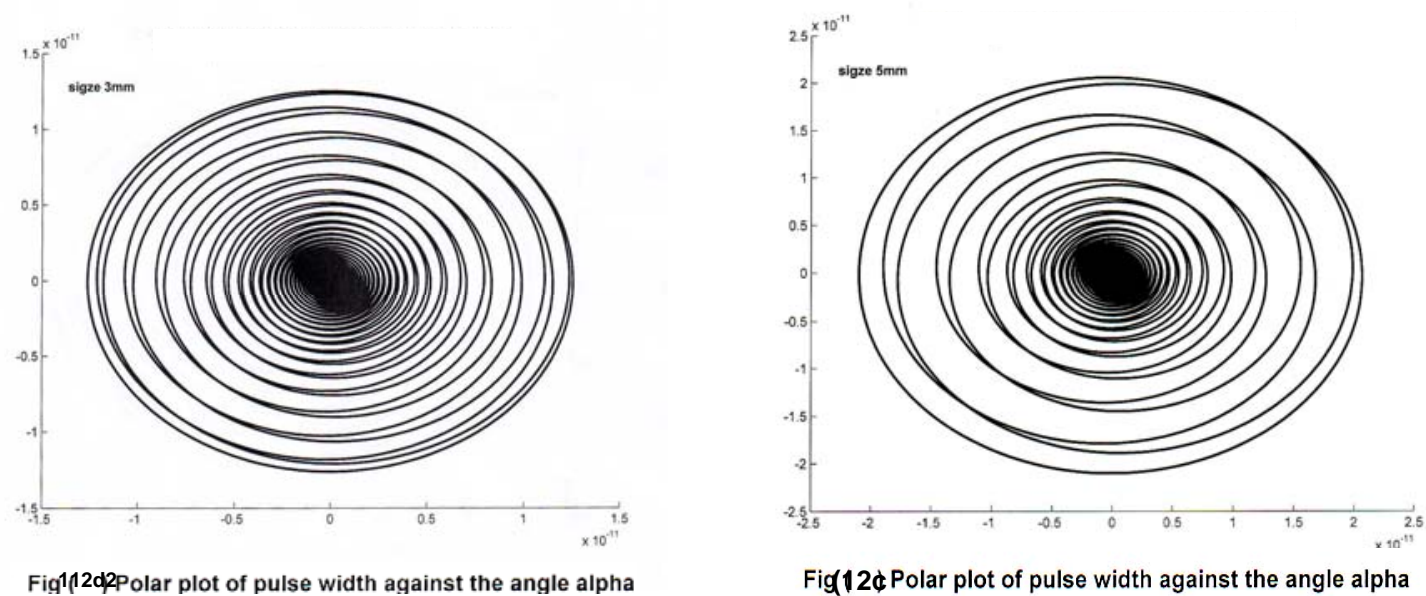

\section{Conclusions}

A short pulse $\mathrm{x}$-ray source is an important tool for studying the dynamics of material in the fundamental time scale.In this simulation, we have shown that the differential cross section of Thomson scattering depends on many parameters specially such as scattering angle, x-ray emitting angle and impact electron energy. Noticeable argument can be drawn from these dependence that the minimum impact energy required to generate short pulse $\mathrm{x}$-ray is about $(0.5 \mu \mathrm{ev})$.Furthermore another conclusion can be illustrated that the projection of $x$-ray pulse length against the scattering angle (alpha) is concentric at ( $\operatorname{sig} \mathrm{ze}=1 \mathrm{~mm}$ ) and deviated apparently bigger than this value.

\section{References}

1) University of Turku, Astrophysics, Lecture II, (2009). Finland.

2) http: \\pbpi.physics-Ucla.edu/Education/Courses/physics-199/Inverse comption.

3) www. Sung sb-edu/iefa 2001/papers/w2-5e/pdf

4) J.Yang, M.Y0r0zu, F.Sakai, T.Yangida, Y.Okaada EPAC 2002. Paris, France.

5) Kuroda D. Ueyama, T. Saito, S. Minaamiguchi, K. Hidume, M. Washio. Proceedings of the 2004 FEL conference, 466.

6) CH.X.Tang, W.H.Huang, H.B. Cheng, Y.Cheng, Q.Du, T.B.Du, Y.CH.Du, XZH, J.F.Hua, G.Huang, Y.Ch.Ge, Y.ZH.Lin, B.Xia, M.J.Xu, X.D.Yuan, SH X.Zheng. Proceedings of 2004 FEL/conference, 622.

7) F.Sakai, J.Yang, M.Yorozu, Y.Okada, T.Yanagida. Proceedings of the 2001/patiele accelerator conference, Chicago.

8) Zelinsky, E. Bulyak, P. Gladkikh, I. Karnaukhov, A. Shcherbakov. Proceedings of APAC 2004, Iuceme, Switzerland.

9) www.thales-Laser.Com.

10) http://en.wikipedia.org/wiki/Thamson-Scattering. 